have been encountered. Therefore burr hole operation may be used with good results as means of simplified surgical approach to chronic subdural hematoma.

Electroencephalographic examinations were done on 14 cases. In 4 cases, generalized seizures or myoclonic discharge were noted from activation by photic stimulation and Bemegride in dosis less than $100 \mathrm{mg}$. The membranes of these hematoma were thin and the thickness of each hematoma was less than $2 \mathrm{~cm}$. These head injuries at the time of trauma were classified into II and III types defined by Araki. Therefore, these data strogly suggest that occurrence of seizures may be derived from organic cerebral damage at the time of trauma rather than from the remaining membrane of hematoma after the operation. However, one patient with thick hematoma membrane had grand mal sezure seven months postoperatively. He has been treated with anticonvulsants, showing no seizures by activation in electroencephalographic examination two years postoperatively. It is only through continued investigation on these problems whether or not there is relation between the remaining membrane of hematoma and occurrence of epilepsy after burr hole operation.

\title{
119. Studies on the Chronic Subdural Hematoma
}

\author{
Shuzo Okumura, Akira Nishimoto,, Tohru Higashi, Keizo Matsumoto, \\ Tasuku MOORI and Shinji TAKEUCHI \\ Department of Neurosurgery, Okayama University Medical School
}

Fourty seven adult and six children subdural hematoma cases were treated at the our service. The adult cases showed the age distribution between 17 to 72 year old, with mode at fourth decade. None of female case was included. 31 out of total 47 cases had history of head injury in previous. The volume of the hematoma mainly stayed in the range between 100 to $150 \mathrm{ml}$. 15 cases were treated by the extirpation of the capsule by craniotomy, and the remainder was treated by the drainage and washing of the hematoma by barr-hole.

The average delay time from the head injury to the starting of the progressive symptoms such as recurrent vomiting, motor weakness, sensory disturbance etc. was one month; and average time from the head injury to the surgical procedure was three months. Those delays were apparently caused by slow swelling of the hematoma. To explanate the swelling, 
Table

\begin{tabular}{l|ccc|cc}
\hline \multicolumn{1}{c|}{ sample } & I & II & III & CSF. & $\begin{array}{c}\text { total } \\
\text { blood }\end{array}$ \\
\hline specific gravity & 1024 & 1026 & 1026 & 1007 & 1026 \\
pH & 7.3 & 7.3 & 7.4 & 7.5 & 7.4 \\
water content & $89 \%$ & $86.8 \%$ & - & - & $80 \%$ \\
ash content & $0.52 \%$ & $0.7 \%$ & - & $0.78 \%$ & $0.74 \%$ \\
osmotic pressure (m osmo) & 295 & 303 & 302 & 290 & 295 \\
protein (g./dl) & 6.5 & 7.0 & 6.5 & 0.02 & 7.3 \\
\hline
\end{tabular}

the content of the subdural hematoma of three cases and CSF. and blood of the first case of these three were studied. (Table)

The result of the analyses made the conclusion that the force of the swelling is colloid swelling power of the protein and not osmotic pressure of the solution. At the first case of analysed three it was calculated that the content of the hematoma was diluted 1.4 times by CSF. depending on the concentration of the protein and ash.

In the six children subdural cases, three was treated by the extirpation of capsule by craniotomy and two of them showed thick capsule membrain with maximum thickness of $1.4 \mathrm{~cm}$. And it was strongly suggested that in the child case, especially with relative long history, removal of the capsule of the hematoma should be thoroughly considered.

\title{
120. Clinical Experiences with Cases of the Chronic Subdural Hematoma
}

\author{
Takao TaKetomo, Shigeo Ueda, Kazuki Sakata, Kunikazu Suhara, \\ Yoshihiro Kawamura, Osamu Sato, Takashi Yamamura, \\ and Yoshitomo KASHIKI \\ Second Surgical Division, Gifu University Medical School
}

In these 8 years 48 cases of the chronic subdural hematoma were treated by us. The ages of the patients ranged from 5 months to 74 years, fifth and sixth decades being predominant. All but 1 case were male. All but 2 cases had histories of head injuries, which were of the type (I) or (II) of Araki (simple loss of consciousness for less than 12 hours) in the majority of the cases. The period between the trauma and appearance of the initial symptoms was 68 days on the average, except 1 case in which 